\title{
Effects of myocardial function in geriatric hypertension at 1 year of follow-up in the single-center SPRINT
}

\author{
Xiaoyan Chen ${ }^{1}$, Qingmei Yang ${ }^{2}$, Jianxiu Fang ${ }^{2}$, and Haifeng Guo ${ }^{3}$ \\ ${ }^{1}$ Affiliation not available \\ ${ }^{2}$ Shanxi Bethune Hospital \\ ${ }^{3}$ Shanxi Medical University
}

December 23, 2020

\begin{abstract}
Background A lower systolic blood pressure (SBP) target reduces major cardiovascular events and mortality from any cause in geriatric hypertension. However, the effect of different SBP targets on left ventricular (LV) function remains unclear. This study aimed to determine changes in LV strain in older hypertensive patients after 1 year of different SBP goals, and to evaluate its effects on LV function in this population. Methods We studied 313 hypertensive adults aged 60 years or older after 1 year of the Systolic Blood Pressure Intervention Trial. They were divided into the intensive group (target SBP: 110-130 mmHg) and the standard group (target SBP: 130-150 mmHg). All participants underwent echocardiography within 1 week after enrollment and 1 year after participating in the study. Global longitudinal strain (GLS) of the LV (endocardial, middle, and epicardial layer: GLS-end, GLS-mid, and GLS-epi, respectively) and improvement of GLS at 1 year ( $\Delta$ GLS-end, $\Delta$ GLS-mid, and $\Delta$ GLSepi) were measured. Results At 1 year, GLS-end in the intensive group was decreased compared with that before the trial $(-23.78 \% \pm 3.10 \%$ vs $-22.58 \%+-3.11 \%, \mathrm{P}<0.05)$. The $\Delta$ GLS-end and $\Delta$ GLS-mid in the intensive group were higher than those in the standard group (both $\mathrm{P}<0.05$ ). Moreover, SBP at 1 year and an angiotensin II type 1 receptor antagonist were independent factors that affected $\triangle$ GLS-end. Conclusions These trial results suggest that a lower SBP target is beneficial for LV myocardial function of older hypertensive patients at 1 year.
\end{abstract}

\section{Hosted file}

manuscript.pdf available at https://authorea.com/users/369642/articles/500389-effects-ofmyocardial-function-in-geriatric-hypertension-at-1-year-of-follow-up-in-the-singlecenter-sprint 

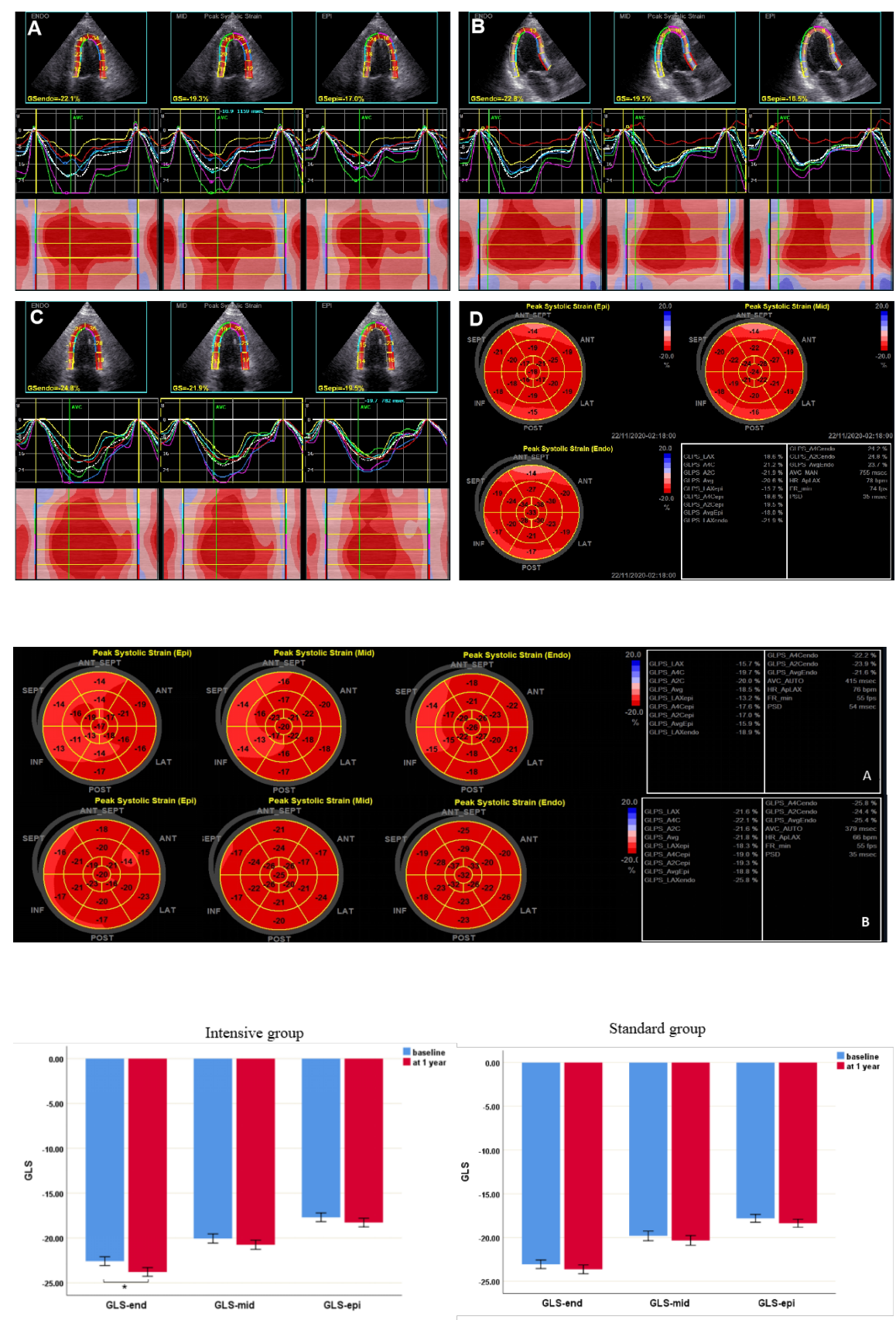


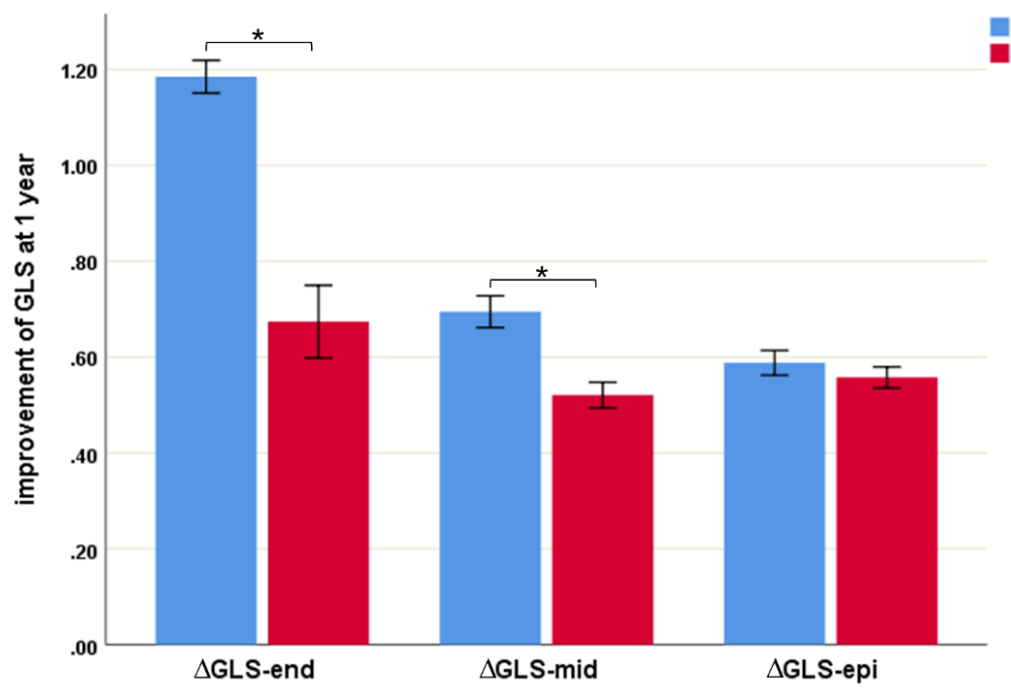

